\title{
The epistemic force of perceptual experience
}

\author{
Susanna Schellenberg
}

(C) Springer Science+Business Media Dordrecht 2013

\begin{abstract}
What is the metaphysical nature of perceptual experience? What evidence does experience provide us with? These questions are typically addressed in isolation. In order to make progress in answering both questions, perceptual experience needs to be studied in an integrated manner. I develop a unified account of the phenomenological and epistemological role of perceptual experience, by arguing that sensory states provide perceptual evidence due to their metaphysical structure. More specifically, I argue that sensory states are individuated by the perceptual capacities employed and that there is an asymmetric dependence between their employment in perception and their employment in hallucination and illusion. Due to this asymmetric dependence, sensory states provide us with evidence.
\end{abstract}

Perceptual experience plays at least three different roles in our lives. It justifies our beliefs about our environment. It brings about conscious mental states. It converts informational input, such as light and sound waves, into representations of features that we attribute to the world. Corresponding to these three roles, there are at least three fundamental questions that have motivated the study of perceptual experience.

Epistemology-question: How does perceptual experience justify our beliefs and yield knowledge of our environment given that perceptual experience can be misleading (we may be subject to illusion or hallucination)?

Mind-question: How does perceptual experience bring about conscious mental states in which our environment appears or seems a certain way to us (irrespective of the way our environment actually is)?

S. Schellenberg $(\square)$

Department of Philosophy, Rutgers University, New Brunswick, NJ, USA

e-mail: susanna.schellenberg@ rutgers.edu 
Information-question: How does a sensory system accomplish the feat of converting varying informational input into mental representations of invariant features that we attribute to the world?

The last decade has seen an explosion of work in philosophy of mind and cognitive science addressing the mind- and information-questions. While there has been fruitful interaction between work on perception in philosophy of mind and cognitive science, there has been much less attempt to integrate this work with issues in epistemology. Theories that are motivated by the mind- and information-questions have to a large extent been developed independently of concerns about how perception provides us with evidence and knowledge of our environment. For the same reason, theories of perceptual experience that are motivated by the epistemology-question have been developed largely independently of concerns about how perceptual experience brings about conscious mental states. ${ }^{1}$ To be sure, most accounts of perceptual justification rely heavily on the idea that perception yields sensory states, which justify beliefs. However, such accounts typically take as a given that such sensory states provide evidence and immediately proceed to addressing the question of what the relationship is between this evidence and relevant beliefs.

There is an increasing recognition that this split has hindered a good understanding of perceptual experience. ${ }^{2}$ Questions in philosophy of mind are intimately connected with questions in epistemology in particular with regard to perceptual experience: arguably the role of experience in yielding sensory states is not independent of its role in justifying our beliefs and yielding knowledge. If this is right, then perceptual experience should be studied in an integrated manner.

The aim of this paper is to make headway in developing a unified account of the phenomenological and epistemological role of perceptual experience. I will argue that experience provides us with evidence in virtue of its metaphysical structure. More specifically, employing will argue that sensory states are individuated by the perceptual capacities employed and that there is an asymmetric dependence between their employment in perception and their employment in hallucination and illusion. Due to this asymmetric dependence, sensory states provide us with evidence.

I will proceed as follows. After briefly mapping the landscape of existing views, I will present a modest externalist account of sensory states. In light of this account of sensory states, I will develop a view of perceptual evidence.

\section{Landscape}

When we perceive a white cup on a desk, it seems to us that there is a white cup on a desk and we are sensorily aware of the white cup on the desk. This is the good case. By definition, when we hallucinate, we are not perceptually related to the external,

\footnotetext{
1 There are notable exceptions, see for instance, Peacocke (1983) and Silins (2005, 2011).

${ }^{2}$ For recent accounts that are sensitive to both the nature of phenomenal character and the structure of perceptual justification, see Hellie (forthcoming) and Silins (forthcoming).
} 
mind-independent object that our experience is seemingly of. So when we suffer a hallucination as of a white cup on a desk, we are not causally related to the white cup our experience is seemingly of. However subjectively our experience can be indistinguishable from a perception: it seems to us that there is a white cup on a desk. This is a kind of bad case. Illusions are a different kind of bad case: when we suffer an illusion it seems to us that there is a property instantiated in our environment, which is not in fact instantiated where it seems to us to be. If perceptions, hallucinations, and illusions can be subjectively indistinguishable, what is the connection between the sensory character of these perceptual states and the subject's perceptual or causal relations to objects and property-instances in her environment?

There are two traditional approaches to answering this question. Common factor views posit that subjectively indistinguishable hallucinations and perceptions share a common element. A subject perceives an object if and only if she is in a mental state that is characterized by this common element and some additional condition obtains. Typically, the additional condition is considered to be a causal relation between the experiencing subject and the perceived object (c.f. Crane 2003; Byrne 2001). This approach is analogous to the view that knowledge can be factorized into belief and some additional element, say justification. By contrast austere relationalists characterize hallucinations in terms of a deficiency of an accurate perceptual experience and argue that perceptions and hallucinations do not share a common element (c.f. Campbell 2002; Martin 2002; Brewer 2006). This approach is analogous to the view that mere belief is to be analyzed as deficient of, but subjectively indiscriminable from knowledge.

With common factor views, I argue that subjectively indistinguishable perceptions, hallucinations, and illusions share a metaphysically substantial, common factor that grounds their sensory character. But with austere relationalists, I argue that hallucinations can only be understood in terms of a deficiency of perceptions. More specifically, I will argue that there is an asymmetric dependence of the bad on the good case. I will show how this way of understanding the metaphysical structure of perceptual experience can be exploited for a view of perceptual evidence.

\section{Modest phenomenological externalism}

To set the stage for the view of sensory states that I will present, I will briefly consider the orthodox view. When a subject sees a white cup, it is natural to say that it seems to her that there is a white cup precisely because she is perceptually related to a white cup. Contrast the perceiver's situation with the situation of someone who is suffering a hallucination. Since the hallucinating subject is not perceptually related to a white cup, the fact that it seems to him that there is a white cup present cannot be explained in terms of an awareness relation to an external, mindindependent object and the properties it instantiates. How then should we explain the sensory character of hallucinations? The standard response is to argue that since hallucinating subjects are aware of something, they must stand in an awareness relation to some thing that accounts for this sensory character. The idea is moreover 
that since a hallucinating subject does not stand in an awareness relation to any external, mind-independent object that it seems to her she is perceiving, she must be standing in an awareness relation to some other kind of object-some kind of peculiar entity. This peculiar entity has been understood to be an abstract entity, such as a property-cluster, an (uninstantiated) universal, or a proposition. It has also been understood to be a strange particular, such as a sense-datum, a quale, or an intentional object. ${ }^{3}$ Sensory character is then understood to be constituted by awareness relations to such peculiar entities.

A very different view of sensory character is to understand it in terms of a mental activity, more specifically in terms of employing perceptual capacities. ${ }^{4}$ The idea is that when we perceive, we employ perceptual capacities by means of which we differentiate and single out particulars in our environment. The relevant particulars are external and mind-independent objects, events, property-instances, and instances of relations. Sensory states are individuated by employing perceptual capacities in a sensory mode, that is, modes such as seeing, hearing, touching, smelling, or tasting.

Consider Percy who perceives a white cup on a desk. He employs his capacity to discriminate white from other colors and to single out white in his environment. Similarly he employs his capacity to differentiate and single out cup-shapes from, say, computer-shapes and lamp-shapes. In virtue of employing such capacities, he is in a sensory state that is of a white cup.

How does this way of understanding the sensory character of perceptions generalize to hallucinations and illusions? To answer this question, let's consider the fact that if we possess a concept, then we can employ it even if we fail to denote anything. After all, if we say "That's a horse" pointing to where in fact there is no horse, we are arguably using the very same concept HORSE that we would be using were we pointing at a horse. The very same thing can be said of perceptual capacities. If we possess a perceptual capacity, then we can employ it even if we are not accurately perceiving. If this is right, then we can say that when we hallucinate, we employ the very same capacities that in a subjectively indistinguishable perception are employed while being perceptually related to external, mind-independent particulars. Since in hallucination, we are not perceptually related to a particular, we fail to single out a particular in our environment. We merely purport to single out a particular. As a consequence, at least some of the perceptual capacities employed are baseless. They are baseless in the sense that the targets of discrimination and selection-external, mind-independent particulars - are absent. Analogously, if we employ concepts, but fail to refer, the concepts employed remain empty.

Consider Hallie who suffers a hallucination as of a white cup on a desk. Like Percy, she employs the capacity to discriminate and single out white from other colors and she employs the capacity to differentiate and single out cup-shapes from, say, computer-shapes and lamp-shapes. Since she is hallucinating rather than

\footnotetext{
${ }^{3}$ For views according to which hallucinating subjects stand in awareness or acquaintance relations to property-clusters, see Johnston (2004); for intentional objects, see Harman (1990), Lycan (1996); for propositions, see Russell (1913); for sense-data, see Robinson (1994); for qualia, see Block (2003); for Meinongian objects, see Parsons (1980).

${ }^{4}$ I developed this view in my 2011.
} 
perceiving and so not perceptually related to a particular white cup, the capacities she employs are baseless. Yet even though she fails to single out any white cup, she is in a sensory state that is as of a white cup in virtue of employing the capacity to discriminate and single out white from other colors and cup-shapes from other shapes.

If it is right that two experiences in which ceteris paribus all the same perceptual capacities are employed in the same sensory mode have the same sensory character, then subjectively indistinguishable perceptions, hallucinations, and illusions will share a metaphysically substantial common factor. The common factor is individuated by the perceptual capacities that the subject employs in a sensory mode.

In order to show how this amounts to an externalist view of sensory character despite positing a common element between subjectively indistinguishable perceptions, hallucinations, and illusions, it will be necessary to specify what it means to possess perceptual capacities. When we perceive or suffer an illusion or hallucination, we employ perceptual capacities the possession of which depends on perceptual relations to the very particulars that the capacities single out in the case of an accurate perception. ${ }^{5}$ We can understand the requirement for possessing perceptual capacities in the following way: to possess a perceptual capacity is to be in a position to single out the particulars that capacity functions to single out.

The perceptual capacities in play can be understood to be discriminatory, selective capacities or concepts-at least on certain ways of understanding concepts. There is good scientific evidence that discriminatory, selective capacities are cognitively the most low-level mental capacities employed in perception, so I will focus on this kind of perceptual capacity. ${ }^{6}$ A discriminatory, selective capacity is a low-level mental capacity that functions to differentiate, single out, and in some cases type the kind of particulars that the capacity is of. For example, if we possess the discriminatory, selective capacity that functions to differentiate and single out red, we are in a position to differentiate instances of red from other colors in our environment and to single out instances of red. More generally, to possess a discriminatory, selective capacity is to be in a position to differentiate and single out the type of particulars that the capacity is of, were one related to such a particular. ${ }^{7}$

\footnotetext{
5 The notion of property-instances in play is best illustrated with an example: when one sees two qualitatively identical white cups, the cups instantiate the same property, but the instances are distinct. When one suffers a hallucination as of a white cup, it seems to one that there is a white cup present, but since one is not perceptually related to the object that it seems to one is present, one is not perceptually related to any instance of whiteness.

${ }^{6}$ For discussions of the role of basic visual capacities and pre-attentive discrimination in early vision, see Julesz (1981), Watson and Robson (1981), Sagi and Julesz (1985), Malik and Perona (1990), Krummenacher and Grubert (2010), and To et al. (2011).

7 The notion of capacity in play can but need not be understood in a teleological, phylogenetic, virtue epistemological, or ontogenetic manner. For such accounts, see Millikan (1989), Neander (1996), Sosa (1991, 2007), Zagzebski (1996), Greco (2001, 2010), and Burge (2003, 2010). As I will argue in the rest of this section, a sensory state provides phenomenal evidence insofar as it is individuated by capacities that are metaphysically and explanatorily dependent on the good case. As I will show, accepting this idea is compatible with accepting that such capacities may more often than not be used in a way that fails to produce accurate representations of the environment.
} 
So if we possess such a capacity, then-assuming no finking, masking, or other exotic case obtains (see Lewis 1997) — the following counterfactual should hold: if we were perceptually related to a particular that the capacity functions to single out, then we would be in a position to single out such a particular. ${ }^{8}$ Singling out a particular is a proto-conceptual analogue of referring to a particular. While referring requires conceptual capacities, singling out particulars requires no such cognitively rich capacities. There are further analogies between discriminatory, selective capacities and concepts. Like concepts the same discriminatory, selective capacity can be employed in different environments and in this sense such capacities are repeatable. Moreover, like concepts the discriminatory, selective capacities can be more or less fine-grained. Say you are seeing a field of flowers that are shades of red and yellow. You can employ your capacity to discriminate between red and yellow and thus be aware of a field of red and yellow flowers. Alternatively, you can employ your capacity to discriminate between crimson, scarlet, and vermillion, and between lemon, mustard, and chartreuse and thus be aware of the colors in front of you in a more fine-grained way.

How does appealing to such capacities help understand sensory states? The suggestion is that sensory states are individuated by employing perceptual capacities in a sensory mode. Any two experiences in which all the same capacities are employed in the same sensory mode will have the same sensory character if all else is equal. Although such capacities are in turn individuated by the type of particulars in the environment that they function to single out, arguably they can be employed even if one is misperceiving or hallucinating. One could be prompted to employ such capacities due to nonstandard circumstances: unusual brain stimulations or misleading distal inputs. If this is right, then we can employ a discriminatory, selective capacity even if a relevant particular is not present-where a relevant particular is a token of the type that the capacity functions to single out. The capacities employed account for the fact that in hallucinations we can purport to single out particulars: from a first-person perspective it can seem as if we were perceptually related to particulars in our environment, even if we are suffering an illusion or a hallucination.

Since sensory states are understood as individuated by employing perceptual capacities rather than the capacities themselves, whether the capacities employed are baseless is not revealed in sensory character. So it is not revealed in our sensory character whether we succeed in differentiating and singling out particulars, and so whether we are perceiving or hallucinating. The important point is that one can distinguish the employment of the capacity-what perceptions, hallucinations, and illusions have in common-from discriminating and singling out a particular-the matter on which perceptions, hallucinations, and illusions differ. It is the

\footnotetext{
8 The inference from a claim about perceptual capacities to a counterfactual fails in finking, masking and similarly exotic cases. However, all the standard ways of fixing the disposition-to-counterfactual inference can be exploited for the capacity-to-counterfactual inference. See in particular Lewis (1997). Finding a formulation of the capacity-to-counterfactual inference that is indefeasible in light of all possible finking, masking, and similarly exotic cases would be a project of its own. Therefore, I will here work on the assumption that no such exotic cases obtain. This assumption is independently plausible.
} 
employment of the capacity that determines the sensory state. Whether or not a particular is singled out does not affect the sensory state.

While standard views analyze sensory states in terms of awareness relations to peculiar entities, the suggested view makes it possible to acknowledge that a hallucinating subject does not stand in an awareness relation to anything despite being in a sensory state that purports to be of mind-independent particulars.

I have argued that sensory character should be analyzed in terms of employing perceptual capacities, however these capacities are in turn analyzed in terms of perceptual relations to external, mind-independent objects and property-instances. Insofar as one's sensory state is individuated by the perceptual capacities employed, sensory states are inherently related to the objects and property-instances that these perceptual capacities single out in the good case. In this sense, they are inherently related to the perceiver's environment.

This approach allows us to recognize the austere relationalist insight that the sensory character of perception can and should be explained in terms of perceptual relations to the very external, mind-independent objects and property-instances of which a perceiver is aware. Austere relationalists argue that the sensory character of a perception should be understood in terms of an awareness or acquaintance relation to particulars in the perceiver's environment. This insight demystifies the sensory character of perception. However, by arguing for the radical thesis that all there is to having an experience with a certain sensory character is to be perceptually related to one's environment, austere relationalists leave mysterious how one could be in a sensory state if one is not perceiving, but rather suffering an illusion or a hallucination. ${ }^{9}$ By introducing perceptual capacities that ground our ability to single out particulars, we can reject this radical thesis. By rejecting the radical thesis, we can give a straightforward explanation of what accounts for the sensory character of illusions and hallucinations. ${ }^{10}$

So against austere relationalists, I have argued that perceptions and hallucinations share a common element that grounds their sensory character. However with austere relationalists, I have argued that hallucinations exhibit a deficiency that can only be explained with reference to accurate perceptions. Hallucinations are derivative of perceptions insofar as the perceptual capacities employed in hallucinations can only be specified with reference to their possible roles in perceptions.

\section{Perceptual evidence}

How does analyzing sensory states in terms of employing perceptual capacities help explain why it is rational to heed the testimony of our senses? As I will argue, sensory states are systematically linked to what they are of in the good case in the

\footnotetext{
9 For a defence of this radical austere relationalist thesis, see Campbell (2002), Brewer (2006), and Fish (2009). Martin (2004) argues for a more moderate version of austere relationalism.

${ }^{10}$ For a detailed analysis of how this view can account for hallucinations of uninstantiated properties, the difference between the sensory character of perceptual experience and thought, phenomena such as blurriness and after-images, and other difficult cases, see my 2011.
} 
sense that the perceptual capacities employed in the bad case are explanatorily and metaphysically parasitic on their employment in the good case. I will then show that in virtue of this systematic linkage, it is rational to heed the testimony of our sensory states.

\subsection{The asymmetric dependence of the bad case on the good case}

There is an explanatory primacy of the good over the bad case since one can give an analysis of the perceptual capacities employed in the bad case only by appealing to their role in the good case. Consider again Hallie who suffers a hallucination as of a white cup on a desk. Even though she fails to single out anything white, she is in a sensory state that is as of an instance of white in virtue of employing the capacity to discriminate and single out white from other colors. She would single out an instance of white, were she in the good case-assuming that no finking, masking, or other exotic case obtains. After all, she is employing a discriminatory, selective capacity the very function of which is to differentiate white from other colors and to single out white in her environment. In this sense, we need to refer to what Hallie would discriminate between and what she would single out in the good case in order to explain the role of the capacities she employs in the bad case.

The explanatory primacy of the good over the bad case is licensed by a more basic metaphysical primacy. There is a metaphysical primacy of the good over the bad case insofar as one can possess the discriminatory, selective capacities employed in the bad case only in virtue of being the kind of being that could employ those very capacities in the good case. Call this the metaphysical primacy thesis. Why should we accept this thesis? The function of discriminatory, selective capacities is to differentiate and single out the type of particulars that the capacity is of. It would be unclear what it would mean to possess a discriminatory, selective capacity, the very function of which is to single out a type of particular, without being in a position to single out such a particular when perceptually related to one. If we possess the capacity to discriminate and single out white from other colors, we can use this capacity to single out white in our environment. Were we not in a position to use our capacity in this way, we would not count as possessing the capacity. In short, while discriminatory, selective capacities can be employed in hallucination, they are individuated by relations between perceivers and their environment insofar as the function of the capacity is to differentiate and single out, say, instances of white in perception. In this sense, there is a metaphysical priority of the good over the bad case. As I will show in the next section, it is in virtue of this metaphysical priority that sensory states provide us with evidence.

The metaphysical priority thesis entails the counterfactual that if we possess a discriminatory, selective capacity, then-assuming that no finking, masking, or other exotic case obtains-we would be in a position to single out a relevant particular, were we related to such a particular. However, it also entails the counterfactual that if we possess such a capacity, we would fail to single out a relevant particular, were we not related to such a particular. While it entails these symmetric counterfactuals, the metaphysical priority thesis is not to be identified with them. After all, the thesis posits that there is an asymmetry between the role of 
the good and the bad case. To motivate this consider the fact that the heart has the function to pump blood. It does not have the function to fail to pump blood-though in the bad case it will fail. One possible way to understand this asymmetry is in terms of evolution: the function of the heart is to do what it was selected for (Millikan 1984). However, it need not be understood in an evolutionary way. Any plausible account of natural function will support the idea that the heart has the function to pump blood rather than the function to fail to pump blood. Likewise, perceptual capacities have the function to single out particulars in the environment. They do not have the function to fail to single out particulars. An evolutionary account of function would posit that perceptual capacities evolved for the purpose of singling out particulars rather than for the purpose of failing to single out particulars. However again, there is no need to explain the asymmetry in evolutionary terms. On any plausible account of natural function, we can say that perceptual capacities function to single out particulars rather than function to fail to single out particulars.

Accepting the metaphysical priority thesis is compatible with acknowledging that one could possess a perceptual capacity that one has never actually used successfully in perception. Moreover, the perceptual capacities employed in hallucinations need not have been acquired through perceptions. They might be innate, they might have been acquired through testimony, or they might have been arrived at through imagination. So the metaphysical priority thesis does not imply that we must have successfully used a perceptual capacity in the past to count as possessing such a capacity. It implies only that we must be in a position to use the capacity successfully when perceptually related to a relevant particular. ${ }^{11}$

\footnotetext{
11 Now, does the existence of a perceptual capacity require the existence of at least one successful employment of that capacity? While it is possible to possess such a capacity without having been perceptually related to any particulars of the type that the capacity singles out in the good case, it is plausible that any such perceptual capacity is grounded in perception insofar as the existence of the capacity depends on perceptions of the particulars that the capacity singles out. If this is right, then it follows that there cannot exist any such perceptual capacity that is not grounded in perception. However, it does not follow from this that an individual subject must have had perceptions of the particulars that the capacity singles out to possess the relevant capacity. It follows only that there can exist a perceptual capacity that functions to single out a type of particular, only if a particular of that type has been perceived by someone, somewhere. The argument for the metaphysical priority of the good over the bad case does not depend on resolving the question of whether the existence of a perceptual capacity requires the existence of at least one successful application by someone, somewhere. However, depending on what stance one takes on this issue one must either reject or accept the metaphysical possibility of the scenario of a world of brains in a vat that can hallucinate. Regardless of what stance one takes on this issue, the suggested view allows that a brain in a vat in our world could have hallucinations and so phenomenal evidence.

Can Swampman possess perceptual capacities? Swampman is a being that came into existence through a bolt of lightning and so has no causal history (Davidson 1987). If perceptual capacities are understood in an evolutionary way, then Swampman could not possess the capacities in play. However, if they are understood in a non-evolutionary way, then Swampman could possess the relevant capacities. After all, no past experiences are necessary to possess such capacities. The condition for their possession is understood counterfactually: if one possess the capacity to single out red, then one would be able to single out an instance of red, were on related to such an instance. For present purposes, we can remain neutral on whether capacities are understood in an evolutionary or a non-evolutionary sense.
} 
I have argued that if a subject's environment sensorily seems a certain way to her (regardless of how it in fact is), then she is in a sensory state that is systematically linked to what it is of in the good case. More specifically, I have argued that sensory states are systematically linked to what they are of in the good case in the sense that the perceptual capacities employed in the bad case are explanatorily and metaphysically parasitic on their employment in the good case. In the next section, I will show how this asymmetric dependence of the bad case on the good case can be exploited for an account of perceptual evidence.

\subsection{Perceptual evidence and systematic linkage}

How do we get from the metaphysical fact that sensory states are systematically linked to what they are of in the good case to the epistemic fact that the sensory state provides us with evidence? I will argue that if a subject is in a sensory state that is systematically linked to what it is of in the good case, then she is in a sensory state that provides perceptual evidence for what the state is of in the good case. The truth of this thesis depends on two principles. The first principle is that if sensory states are systematically linked to what they are of in the good case in the sense specified, then it is epistemically rational to heed the testimony of these sensory states. The second principle is that if it is epistemically rational to heed the testimony of sensory states, then they provide evidence. I will give support to each principle in turn.

I argued that sensory states are systematically linked to particulars of the type that the sensory state is of in the good case insofar as it is the function of the perceptual capacities that determine the sensory state to single out the relevant particulars. If it is the function of a perceptual capacity to single out, say, instances of red and employing that perceptual capacity yields sensory states of red, then it is rational to heed that sensory state and so trust that something red is present. In speaking of it being the function of discriminatory, selective capacities to single out what they are of in the good case, I do not mean to speak of their actual reliability but rather of how they are to be understood metaphysically. It is the function of a discriminatory, selective capacity to single out, say, instances of red. This is so regardless of how often the capacity in fact singles out instances of red. The second principle states a sufficient condition for something to count as evidence. It follows from a substantive but largely uncontroversial view about evidence, namely, that it is a crucial property of evidence that if it is epistemically rational to heed $x$ in the absence of defeaters, then $x$ provides evidence. ${ }^{12}$

\footnotetext{
12 See Ayer (1972), Kelly (2003, 2007), Neta (2003, 2008), Weatherson (2005), and Pryor (forthcoming) for discussions of this property of evidence. An interesting question is what the connection is between the strength of the evidence we have for a proposition and our confidence in that proposition. For discussion of the relation between having evidence for $p$ and having confidence in $p$, see Neta $(2003,2008)$ and Silins (2005). Since our concern here is restricted to the questions of what evidence perceptual experience provides us with and why it is rational to heed it, we can bracket this issue for the purposes of this paper. I reserve a detailed discussion of how the account developed here connects to questions about confidence for a future paper.
} 
Now one might object that beliefs are linked to what they are of in the good case, but it is not rational to treat beliefs as evidence. So why is it rational to treat sensory states as evidence but not beliefs ${ }^{13}$ In response, we can concede that many things are in some way linked to what they are of in the good case. It is not rational to treat all those things as evidence. However, I argued that sensory states are systematically linked to what they are of in the good case in the sense that the perceptual capacities employed in the bad case are explanatorily and metaphysically parasitic on their employment in the good case. So the systematic linkage between sensory states and what they are of in the good case was understood in a specific way. The very nature of perceptual capacities is determined by functional connections between perceivers and their environment. The capacities employed in perception link perceptual states with particulars in the environment. Perception is our original connection to our environment. The capacities that determine beliefs are arguably not systematically linked to what they are of in the good case in this way. After all, while the capacities that determine beliefs function to get the facts right, they do not generally function to single out particulars. Therefore, the argument provided for why it is rational to heed the testimony of our senses does not over-generalize to beliefs. ${ }^{14}$

\subsection{Wider context}

I have argued that our perceptual evidence in the bad case is brought about by employing the very same perceptual capacities that in the good case allow us to perceptually navigate our environment. While these capacities are individuated by functional relations to the particulars they single out in perception, we can employ the same capacities while failing to single out a relevant particular. So having perceptual evidence is compatible with our perceptual capacities being employed baselessly. As a consequence, hallucinations provide us with tangible, though misleading evidence. The evidence is individuated by our sensory states. So it is a kind of phenomenal evidence. A subjectively indistinguishable perception will provide us with the very same phenomenal evidence. After all, our sensory state can be the very same regardless of whether we are perceiving or hallucinating. For the purposes of this paper, we can remain neutral on whether we have any additional factive evidence in the case of an accurate perception. ${ }^{15}$

While the developed notion of evidence is externalist in that phenomenal evidence is individuated by perceptual capacities employed and there is an asymmetric dependence of the capacities employed in the bad case on their

\footnotetext{
13 This objection is due to Alex Byrne.

14 Now, what if we assume for the sake of argument both that beliefs are a kind of sensory state and that the capacities that determine beliefs are explanatorily and metaphysically parasitic on their employment in the good case? On these two controversial assumptions, it is plausible that beliefs provide us with evidence. So on these assumptions, the argument provided for why it is rational to heed the testimony of our senses generalizes to beliefs. It does not however over-generalize and so would not be a problem for the developed view, since beliefs are now understood to have many of the fundamental properties of perceptual states.

15 For a defense of the view that perception provides us with both phenomenal and factive evidence, see my forthcoming.
} 
employment in the good case, we can have phenomenal evidence even when we are in the bad case. The developed notion of phenomenal evidence is internalist only insofar as the phenomenal evidence of two experiencers in different environments can be the very same. It is not internalist regarding the accessibility of the evidence: it does not impose any accessibility requirement on evidence. More importantly, it is not internalist insofar as our phenomenal evidence is understood in terms of an asymmetric dependence of the bad case on the good case. ${ }^{16}$

This externalist notion of phenomenal evidence makes room for the idea that having evidence is a matter of being in an epistemic position that is well situated to tell us the truth, while allowing that we can have such evidence even if we happen to have been led astray and so are in a state that is not accurate with regard to our environment. As a consequence, the suggested view shows how experience provides us with phenomenal evidence even in the bad case without retreating to introspective evidence.

The developed view about perceptual evidence makes room for an externalist account of the epistemic role of perceptual experience that does not depend on and does not entail reliabilism (Goldman 1979). One might argue that it is in virtue of perceptual capacities being reliable that the sensory states they determine provide us with evidence. On the defended view, sensory states provide us with evidence since sensory states are systematically linked to what they are of in the good case. I argued that sensory states are systematically linked to what they are of in the good case in the sense that the perceptual capacities employed in the bad case are explanatorily and metaphysically parasitic on their employment in the good case. So the notion of systematic linkage in play is understood in terms of a metaphysical and explanatory primacy notion rather than a reliabilist notion. Indeed, the perceptual capacities that determine sensory states may more often than not be used in ways that fail to produce accurate representations of the world. So such capacities may fail to be reliable. But while the suggested view does not depend on and does not entail reliabilism, it is compatible with such a view.

The suggested view of phenomenal evidence is compatible not only with reliabilism, it is compatible also with the basic commitments of virtue epistemology. ${ }^{17}$ However again one can accept the view without accepting the basic commitments of virtue epistemology. The suggested view for instance neither entails nor depends on the thesis that epistemology is a normative discipline. Neither the capacities nor the metaphysical and explanatory primacy notions in play need be understood in terms of virtues or any other normative notion.

\section{Conclusion}

I have argued for an externalist view of perceptual evidence that makes room for a phenomenal conception of evidence. My explanation for why sensory states provide

\footnotetext{
16 See Pryor (2001, pp. 105-108) for useful distinctions between ways of understanding the access requirement on our evidence and more generally different forms of epistemic internalism.

17 For virtue epistemological approaches, see among others Sosa (1991), Zagzebski (1996), and Greco (2001).
} 
phenomenal evidence is that the perceptual capacities employed in the bad case are systematically linked to their employment in the good case insofar as we can possess the capacities employed in the bad case only in virtue of being the kind of being that could employ the relevant capacities in the good case. So the perceptual capacities employed in the bad case are metaphysically and explanatorily parasitic on their employment in the good case. There is a metaphysical primacy of the good over the bad case insofar as one can possess the perceptual capacities only in virtue of being the kind of being that could successfully employ those capacities in the good case. There is an explanatory primacy of the good over the bad case insofar as giving an analysis of the perceptual capacities employed in the bad case requires appealing to their role in the good case.

\section{References}

Ayer, A. J. (1972). Probability and evidence. New York: Columbia University Press.

Block, N. (2003). Mental paint. In M. Hahn \& B. Ramberg (Eds.), Reflections and replies: Essays on the philosophy of Tyler Burge. Cambridge, MA: MIT Press.

Brewer, B. (2006). Perception and content. The European Journal of Philosophy, 14, 165-181.

Burge, T. (2003). Perceptual entitlement. Philosophy and Phenomenological Research, 67, 503-548.

Burge, T. (2010). Origins of objectivity. Oxford: Oxford University Press.

Byrne, A. (2001). Intentionalism defended. The Philosophical Review, 110, 199-240.

Campbell, J. (2002). Reference and consciousness. Oxford: Oxford University Press.

Crane, T. (2003). The intentional structure of consciousness. In A. Jokic \& Q. Smith (Ed.), Consciousness: new philosophical perspectives. Oxford: Oxford University Press.

Davidson, D. (1987). Knowing one's own mind. Proceedings and Addresses of the American Philosophical Association, 60, 441-458.

Fish, W. (2009). Perception, hallucination, and illusion. New York: Oxford University Press.

Goldman, A. (1979). What is justified belief. In G. Pappas (Ed.), Knowledge and justification. Dordrecht: Reidel.

Greco, J. (2001). Virtues and rules in epistemology. In A. Fairweather \& L. Zagzebski (Eds.), Virtue epistemology. Oxford: Oxford University Press.

Greco, J. (2010). Achieving knowledge: A virtue-theoretic account of epistemic normativity. Cambridge: Cambridge University Press.

Harman, G. (1990). The intrinsic quality of experience. In J. Tomberlin (Ed.), Philosophical perspectives (Vol. 4). Northridge: Ridgeview Publishing Company.

Johnston, M. (2004). The obscure object of hallucination. Philosophical Studies, 120, 113-183.

Julesz, B. (1981). A theory of preattentive texture discrimination based on first-order statistics of textons. Biological Cybernetics, 41, 131-138.

Kelly, T. (2003). Epistemic rationality as instrumental rationality: A critique. Philosophy and Phenomenological Research, 66, 612-640.

Kelly, T. (2007). Evidence and normativity: Reply to Leite. Philosophy and Phenomenological Research, $75,465-474$.

Krummenacher, J., Grubert, A., et al. (2010). Inter-trial and redundant-signals effects in visual search and discrimination tasks: Separable pre-attentive and post-selective effects. Vision Research, 50, 1382-1395.

Lewis, D. (1997). Finkish dispositions. Philosophical Quarterly, 47, 143-158.

Lycan, W. G. (1996). Consciousness and experience. Cambridge, MA: MIT Press.

Malik, J., \& Perona, P. (1990). Preattentive texture discrimination with early vision mechanisms effects. Journal of the Optical Society of America, 7, 923-932.

Martin, M. G. F. (2002). The transparency of experience. Mind and Language, 17, 376-425.

Martin, M. (2004). The limits of self-awareness. Philosophical Studies, 103, 37-89.

Millikan, R. G. (1984). Language, thought and other biological categories. Cambridge, MA: MIT Press. 
Millikan, R. G. (1989). In defense of proper functions. Philosophy of Science, 56, 288-302.

Neander, K. (1996). Swampman meets Swampcow. Mind and Language, 11, 118-129.

Neta, R. (2003). Contextualism and the problem of the external world. Philosophy and Phenomenological Research, 66, 1-31.

Neta, R. (2008). What evidence do you have? British Journal for the Philosophy of Science, 59, 89-119.

Parsons, T. (1980). Nonexistent objects. New Haven: Yale University Press.

Peacocke, C. (1983). Sense and content: Experience, thought, and their relations. Oxford: Oxford University Press.

Pryor, J. (2001). Highlights of recent epistemology. British Journal for the Philosophy of Science, 52, 95-124.

Pryor, J. (forthcoming) When warrant transmits. In A. Coliva (ed.), Wittgenstein, epistemology and mind: Themes from the philosophy of Crispin Wright. Oxford: Oxford University Press.

Robinson, H. (1994). Perception. London: Routledge.

Russell, B. (1992). 1913: Theory of knowledge. London: Routledge.

Sagi, D., \& Julesz, B. (1985). Detection versus discrimination of visual orientation. Perception, 14, 619-628.

Schellenberg, S. (2011). Ontological minimalism about phenomenology. Philosophy and Phenomenological Research, 83, 1-40.

Schellenberg, S. (forthcoming). Experience and evidence. Mind.

Silins, N. (2005). Deception and evidence. In Philosophical perspective, epistemology (Vol. 19). Malden, MA: Blackwell Publishers.

Silins, N. (2011). Seeing through the 'Veil of Perception'. Mind, 120, 329-367.

Sosa, E. (1991). Knowledge in perspective. Cambridge: Cambridge University Press.

Sosa, E. (2007). A virtue epistemology: Apt belief and reflective knowledge. New York: Oxford University Press.

To, M. P., Gilchrist, I. D., et al. (2011). Discrimination of natural scenes in central and peripheral vision. Vision Research, 51, 1686-1698.

Watson, A., \& Robson, J. (1981). Discrimination at threshold: Labelled detectors in human vision. Vision Research, 21, 1115-1122.

Weatherson, B. (2005). Scepticism, rationalism and empiricism. In T. Gendler \& J. Hawthorne (Eds.), Oxford studies in epistemology (Vol. 1). Oxford: Oxford University Press.

Wright, C. (2007). The perils of dogmatism. In S. Nuccetelli \& G. Seay (Eds.), Themes from G.E. Moore: New essays in epistemology and ethics. Oxford: Oxford University Press.

Zagzebski, L. T. (1996). Virtues of the mind. Cambridge: Cambridge University Press. 